Article | Special Issue

\title{
Ang SMisasyon ng Lipunang Pinoy
}

\section{Franz Giuseppe F. Cortez}

\begin{abstract}
Taking its cue from phenomena called McDonaldization, Disneyfication, Wal-Martization, and the like, SMization is a neologism derived from the acronym "SM" that stands for Shoe Mart. SM is a business conglomerate in the Philippines and is deemed to be the most successful Filipino business enterprise. By SMization, I refer to the effective perpetuation and fortification of the neoliberal process in the Philippines and the broadening of its effect in all aspects of the lives of Filipinos: economic, social, religious, political, cultural, psychological, moral, ecological, and others. The SM conglomerate symbolizes the successful and effective entry of neoliberalism in the country. SMization may have many features but on this preliminary investigation, I will only explore three phenomena: hyperconsumerism, survival-of-the-fittest culture, and myth of upward mobility.
\end{abstract}

Keywords: SMization, neoliberalism, consumerism, survival-of-thefittest

Nakakainip ang ganitong buhay

Nakakainis ang ganitong buhay

Nakakabaliw ang ganitong buhay

Di nakakaaliw ang ganitong buhay

-Yano (isang bandang Pinoy), "Esem" (Yano, 1994)

\section{Panimula}

$\mathrm{U}$ sung-uso na talagang gawing pandiwa ang maraming makabagong pangngalan: Google at googling, YouTube at youtubing, Facebook at facebooking. Subalit bago pa naging palasak ang mga ito, sumikat na noong dekada ' 80 ang salitang McDonaldization nang ilathala noong 1983 ng Amerikanong sosyolohistang si George Ritzer ang kanyang sanaysay na "The 
McDonaldization of Society." ${ }^{1}$ Gamit ang kaisipan ng Alemang pilosopo na si Max Weber, sinabi ni Ritzer na tumutukoy ang McDonaldization sa patuloy na paglawak ng instrumental na rasyonalidad-isang rasyonalidad na nagbibigay-diin sa kahusayan ng trabaho (efficiency), kasiguruhan at maaasahang resulta nito (predictability), katiyakan (calculability), at kontrol (control). ${ }^{2}$ Pagkatapos mailathala ang sanaysay ni Ritzer, naging palasak na rin ang mga salita tulad ng Disneyization at Disneyfication, ${ }^{3}$ Wal-Martization, ${ }^{4}$ Las Vegasization, ${ }^{5}$ Coca-Colonization, ${ }^{6}$ at eBayization. ${ }^{7}$ Sa papel na ito, isasama ko na rin sa hanay ang isang penomenon sa Pilipinas na tatawagin kong SMization o SMisasyon. ${ }^{8}$

Bagama't marami na ang nag-aaral tungkol sa mall culture sa larangan ng media studies at consumer studies, hindi pa rin kalabisang ituloy ang pagsusuri tungkol dito lalo na sa konteksto ng pinagpanibagong anyo ng Kapitalismo. Maituturo siguro sina Dong Abay ng bandang Yano at Rolando Tolentino na isang guro naman sa Unibersidad ng Pilipinas bilang mga tagahawan ng gawaing-pagsusuri ng malling phenomenon sa bansa. Sa isang awit ni Abay na may pamagat na "Esem," pinuna na niya ang magkahalong aliw at lungkot, salat at sagana, na nararanasan ng mga Pilipino sa tuwing papasok sa SM. Bahagi ng awit ang sumusunod na mga linya:

\footnotetext{
${ }^{1}$ George Ritzer, "The McDonaldization of Society," The Journal of American Culture 6 (1983), 100-107. Pagkatapos ng sampung taon, inilathala ni Ritzer ang aklat na may parehong pamagat.

2 Tingnan sa George Ritzer, The McDonaldization of Society: An Investigation into the Changing Character of Contemporary Social Life (California: Pine Forge, 1993).

${ }^{3}$ Sinabi ni A. E. Bryman na magkaiba raw ang Disneyfication at Disneyization. Tingnan sa A. E. Bryman, “The Disneyization of Society,” The Sociological Review, 47: 1 (1999), 2547.

4 "... a change in the social relations of production where power shifts from suppliersmanufacturers to giant retailers, with the former trickling insecurity downwards to their flexible workforce in their search for disciplinary low-cost strategy." Daryl Reed, Peter Utting, and Ananya MukherjeeReed, eds., Business Regulations and Non-State Actors: Whose Standards, Whose Development? (London: Routledge, 2012), 178.

${ }^{5}$ Gamit ang kaisipan ni Neil Postman, sinabi ni Aaron Duncan na ang Las Vegasization ay tumutukoy sa unti-unting pagkahirati ng lipunan sa glamor at mga nagkikislapang ilaw, sa pustahan at sugal, at sa pangangailangan na laging dapat ay inaaliw at naaaliw. Aaron Duncan, Gambling with the Myth of American Dream (New York: Routledge, 2015).

${ }^{6}$ Reinhold Wagnleitner, Coca-Colonization and the Cold War: The Cultural Mission of the United States in Austria after the Second World War (London: University of North Carolina, 1994).

7 Vladimir Madik, Aaron Ahuvia, and Elif Izberk-Bilgin, "Ebayization," in The Blackwell Encyclopedia of Sociology, ed. by George Ritzer (USA: Blackwell, 2007).

8 Noong 2014, tiningnan ko sa internet kung meron nang gumamit ng salitang SMization. Wala akong nakita. Ang nakita ko ay ang salitang "smize" na ang ibig sabihin ay "to smile with your eyes." Itinuturo raw ito sa mga nagmo-modelo: hindi lamang ngiti ng labi kundi mga matang ngumingiti rin. Walang kaugnayan dito ang nabuo kong salita na SMization na una kong binanggit sa isang panayam noong 2014. (Binasang papel bilang reaksyon sa panayam ni Antonio M. Aureada, "St. Thomas and Education," Pope Leo XIII Lecture Series (University of Santo Tomas, February 2014).
}

(c) 2019 Franz Giuseppe F. Cortez https://www.kritike.org/journal/special issue/cortez april2019.pdf ISSN 1908-7330

(c) BY-NC-ND 


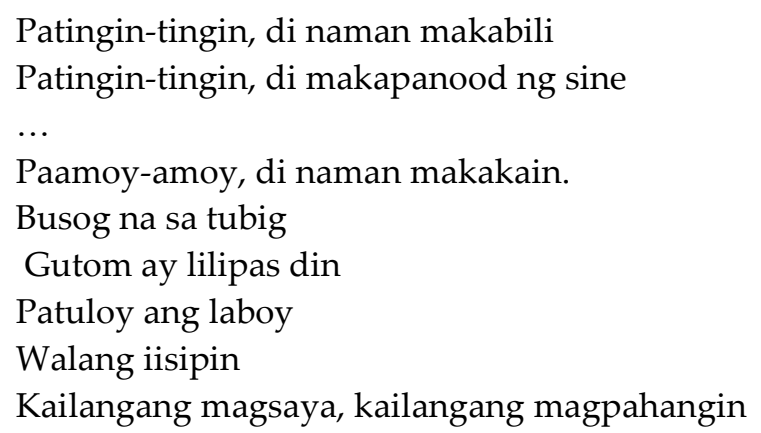

Sa isang aklat naman tungkol sa kulturang popular, tinalakay at sinuri ni Tolentino ang pulitikang bumabalot sa mga penomenon katulad ng computer games, skin whitener, droga, at mall. Bagama't ang pagtangkilik ng mga Pilipino sa mall ay nagdudulot ng maraming kasiyahan, ipinaliwanag ni Tolentino na kaakibat din nito ang kasawian. ${ }^{9}$ Ipinagpatuloy ni Tolentino ang masusing pag-aaral ukol sa kultura ng mall sa Pilipinas sa kanyang aklat na Kulturang Mall. ${ }^{10}$

Itong kasalukuyang sanaysay ay isa na ring pagpupugay, pagpapatuloy, at pagpapasigla ng mga kaisipan nina Abay at Tolentino. Susuriin ko kung paanong nagkaroon ng ugnayan ang ideolohiya ng neoliberalismo at ang pagdanas ng mga Pilipino sa SM.

\section{Ano ang Neoliberalismo?}

Ayon kay David Harvey, mula pa noong dekada '70 bumaling na ang maraming mga lipunan patungo sa neoliberalistang pag-iisip at praktis. ${ }^{11}$ Noong 1999, binanggit ni Robert McChesney na ang neoliberalismo ang namamayaning politikal at ekonomikong teorya ng ating panahon. ${ }^{12}$ Sinabi naman nina Saad-Filho at Johnston na nabubuhay tayo ngayon sa panahon ng neoliberalismo. ${ }^{13}$ Samantalang sa palagay ni Perry Anderson, ito na nga ang pinakamatagumpay na ideolohiya sa kasaysayan ng daigdig. ${ }^{14}$ Dagdag

\footnotetext{
${ }^{9}$ Rolando Tolentino, Sa Loob at Labas ng Mall Kong Sawi / Kaliluha'y Siyang Nangyayaring Hari: Ang Pagkatuto at Pagtatanghal ng Kulturang Popular (Quezon City: University of the Philippines, 2001).

${ }^{10}$ Rolando Tolentino, Kulturang Mall (Manila: Anvil, 2004).

11 David Harvey, A Brief History of Neoliberalism (Oxford: Oxford University Press, 2005), 2.

${ }^{12}$ Robert McChesney, Introduction to Noam Chomsky, Profit over People: Neoliberalism and Global Order (New York: Seven Stories, 1999), 7.

${ }^{13}$ Alfredo Saad-Filho and Deborah Johnston, Introduction to Neoliberalism: A Critical Reader, ed. by Alfredo Saad-Filho and Deborah Johnston (London: Pluto, 2005), 1.

${ }_{14}$ Perry Anderson, “Renewals," New Left Review 1 (January to February 2000), 17.
}

(C) 2019 Franz Giuseppe F. Cortez https://www.kritike.org/journal/special issue/cortez april2019.pdf ISSN 1908-7330 
pa ni Edward Said, nilamon na nga raw ng neoliberalismo ang daigdig kaakibat ang dulot nitong panganib sa demokrasya at kalikasan. ${ }^{15}$ Sa palagay ng maraming kritiko, ito rin ang dahilan kung bakit patuloy na lumalaki ang agwat ng kawalan ng pagkakapantay-pantay sa pagkamit ng iba't ibang uri ng kapital-kultural, ekonomiko, sosyal, politikal, at enbayronmental. ${ }^{16}$

Hindi madaling bigyan ng ganap na pakahulugan ang neoliberalismo. ${ }^{17}$ Tinuran ni Wendy Larner na maaaring tingnan ang neoliberalismo bilang polisiya, ideolohiya, at pamamahala (governmentality). ${ }^{18}$ Maaari ngang tumukoy ang neoliberalismo sa napakaraming bagay, kaisipan, proseso, programa, polisiya, at resulta. ${ }^{19}$

Walang dudang ang salitang-ugat ng neoliberalismo ay "liberalismo." Nakaugat ito sa classical liberal economics nina Adam Smith at David Ricardo noong ika-18 siglo. Sa pananaw ng mga ekonomistang ito, mabuti ang Kapitalismo bilang isang sistemang ekonomiko na nakabatay sa laissez-faire at malayang merkado. Hindi raw dapat nakikialam ang pamahalaan sa pagpapatakbo ng ekonomiya sapagkat merong mga sariling batas-likás ang merkado at merong "di-nakikitang kamay" na sapat na upang maging maayos ang takbo ng larangang ekonomiko. ${ }^{20}$ Pagkatapos ng napakahabang panahong pamamayagpag ng classical liberal economics, pinagdudahan at tinalikuran ito nang bumagsak ang merkado noong 1929 na nagdulot ng Great Depression. Namayani ang ekonomikong kaisipan ni John Maynard Keynes, ang utak sa likod ng New Deal at ng makabagong welfare state o social democratic state. Sa sistemang Keynesian, itinataguyod ang malaking papel ng pamahalaan sa pamamahagi ng yaman ng lipunan at sa pangangasiwa ng ekonomiya. ${ }^{21}$ Subalit nang magkaroon ng krisis ang Keynesian economics noong dekada '70, muling nabuhay, nanumbalik, at sumigla ang mga pinagpanibagong saligan ng classical liberal economics sa tulong ng mga kaisipan nina Ludwig von Mises, Friedrich Hayek, at Milton

15 Edward Said, "Problems of Neoliberalism," in ZNet (20 September 2000), $<\underline{\text { https://zcomm.org/zcommentary/problems-of-neoliberalism-by-edward-said/>. }}$

${ }^{16}$ Kevin Ward and Kim England, "Introduction: Reading Neoliberalization," in Neoliberalization: States, Networks, Peoples, ed. by Kim England and Kevin Ward (USA: Blackwell, 2007), 2.

17 Philip Mirowski, “Postface: Defining Neoliberalism," in The Road from Mont Pelerin: The Making of Neoliberal Thought Collective, ed. by Philip Mirowski and Dieter Plehwe (Cambridge: Harvard University Press, 2009), 421.

18 Wendy Larner, "Neo-liberalism: Policy, Ideology, Governmentality," Studies in Political Economy, 63:1 (Autumn 2000).

19 Ward and England, "Introduction: Reading Neoliberalization," 11.

${ }^{20}$ Manfred Steger and Ravi Roy, Neoliberalism: A Very Short Introduction (Oxford: Oxford University Press, 2010), 2-3.

${ }^{21}$ Naomi Klein, The Shock Doctrine: Rise of Disaster Capitalism (New York: Metropolitan, 2007), 17.

(c) 2019 Franz Giuseppe F. Cortez https://www.kritike.org/journal/special issue/cortez april2019.pdf ISSN 1908-7330

(c) BY-NC-ND 
Friedman. ${ }^{22}$ Ito na nga ang tinatawag na neoliberalismo na agad nagkaroon ng kongkretong mukha sa mga polisiya nina Auguste Pinoche ng Chile, Ronald Reagan ng Estados Unidos, at Margaret Thatcher ng Gran Britanya. ${ }^{23}$

Bilang isang polisiya, tinatalikuran ng neoliberalismo ang welfare state habang mas masigasig na isinusulong ang malayang merkado. Sabi nga, "Markets good. Government bad." 24 Kaakibat nito, inililipat ang mga pag-aaring pampubliko patungo sa pribadong sektor. Nakabatay ito sa paninindigang mas mahusay ang merkado kaysa gobyerno sa pagtugon sa mga pangangailangan ng tao, pagtamo ng ekonomikong kaunlaran, at pagtaguyod ng kagalingang panlipunan. ${ }^{25}$ Bumabandila ang mga konsepto at polisiyang pribatisasyon, deregulasyon, liberalisasyon, malayang kalakalan, at pribadong pag-aari. Ito raw ang pinakamabisang kasagutan sa paglutas ng mga suliranin ng daigdig. Ganito ang paglalarawan ni Harvey:

Neoliberalism is in the first instance a theory of political economic practices that proposes that human well-being can best be advanced by liberating individual entrepreneurial freedoms and skills within an institutional framework characterized by strong private property rights, free markets, and free trade. ${ }^{26}$

Para sa mga nagtataguyod ng neoliberalismo, mas mapabubuti raw ang lipunan at ang sangkatauhan kung bibigyang-laya ang bawat indibidwal sa pamamagitan ng pagpapaigting ng kanilang mga karapatan sa pribadong pag-aari at pagpapalakas ng isang merkadong malaya sa regulasyon ng pamahalaan. Itinataguyod daw nito ang kalayaan ng bawat isa, ang pagkakapantay-pantay at ang pangako ng kaginhawahan at pag-unlad. Makabubuti sa lahat ng tao na bigyan ng ganap na kalayaan ang merkado at huwag itong pakialaman at pangasiwaan ng pamahalaan. ${ }^{27}$

Subalit ang neoliberalismo ay hindi lang naman patungkol sa buhayekonomiko na may kinalaman sa produksiyon, distribusyon, at pagbili ng

\footnotetext{
${ }^{22}$ Simon Clarke, "The Neoliberal Theory of Society," in Neoliberalism: A Critical Reader, 58.

${ }^{23}$ Daniel Jones, Masters of the Universe: Hayek, Friedman and the Birth of Neoliberal Politics (New Jersey: Princeton University Press, 2012).

${ }^{24}$ Campbell Jones, Rene ten Bos, and Martin Parker, For Business Ethics: A Critical Approach (London: Routledge, 2005), 100.

${ }^{25} \mathrm{~J}$. McCarthy, "Privatizing Conditions of Production: Trade Agreements as Neoliberal Environmental Governance," in Neoliberal Environments: False Promises and Unnatural Consequences, ed. by N. Heynen, J. McCarthy, S. Prudham, and P. Robbins (London: Routledge, 2007).

${ }^{26}$ Harvey, A Brief History of Neoliberalism, 2.

${ }^{27}$ Jones, ten Bos, and Parker, For Business Ethics, 96.
}

(c) 2019 Franz Giuseppe F. Cortez https://www.kritike.org/journal/special issue/cortez april2019.pdf ISSN 1908-7330 
mga kalakal. Sa mas malawakang pananaw, ipinapalagay na ang merkado ay siyang angkop na gabay sa lahat ng mga gawain at aspekto ng buhay-tao. ${ }^{28}$ Kung kaya, tumutukoy rin ito sa mas malawak na aspekto at kontekstong kultural, politikal, at sosyal. ${ }^{29}$ Sinabi ni McChesney: "It is precisely in its oppression of nonmarket forces that we see how neoliberalism operates not only as an economic system, but as a political and cultural system as well." 30 Mas nagbibigaylinaw ang pahayag ni Giroux:

Central to its philosophy is the assumption [that] the market drives not just the economy but all of social life. It construes profit-making as the essence of democracy and consuming as the only operable form of agency. It redefines identities, desires and values through a market logic that favors self-interest, a survival-of-the-fittest ethos and unchecked individualism. Under neoliberalism, life-draining and unending competition is a central concept for defining human freedom. ${ }^{31}$

Isa ang Pilipinas sa mga bansang tinukoy bilang guinea pig ng eksperimentong neoliberal.32 Sa obserbasyon nina Broad at Cavanagh, mahabang panahon na na isa ang Pilipinas sa mga "poster child[ren] of an open economy." 33 Noong mga huling taon ng dekada "70 sa ilalim ng rehimen ni Ferdinand Marcos, isinagawa na nga ang structural adjustment program (SAP) sa pangunguna at pamamahala ng tinatawag ni Richard Peet na unholy trinity: World Bank, International Monetary Fund, at World Trade Organization. ${ }^{34}$ Nakapaloob sa SAP ang pangakong pagpapautang sa Pilipinas kapalit ang isang kondisyon: "submit your economies to international market forces. And that

\footnotetext{
${ }^{28}$ Henry Giroux, "Neoliberal Fascism and the Echoes of History," in Truthdig (2 August 2018), < https://www.truthdig.com/articles/neoliberal-fascism-and-the-echoes-of-history/>.

${ }^{29}$ Jennifer Lawn and Chris Prentice, "Introduction: Neoliberal Culture/The Cultures of Neoliberalism," Sites: New Series, 12:1 (2015), 8.

${ }^{30} \mathrm{McChesney,} \mathrm{Introduction} \mathrm{to} \mathrm{Chomsky,} \mathrm{Profit} \mathrm{Over} \mathrm{People,} 9$.

${ }^{31}$ Giroux, "Neoliberal Fascism and the Echoes of History."

32 Timothy Mitchell, "How Neoliberalism Makes Its World," in The Road from Mont Pelerin: The Making of Neoliberal Thought Collective, 393; Walden Bello, "How neoliberalism killed the Philippines' EDSA Republic," in Green Left Weekly (24 June 2016), $<\underline{\text { https://www.greenleft.org.au/content/walden-bello-how-neoliberalism-killed-philippines- }}$ edsa-republic $>$.

33 Robin Broad and John Cavanagh, "Reframing Development in the Age of Vulnerability: From Case Studies of the Philippines and Trinidad to New Measures of Rootedness," Third World Quarterly, 32:6 (2011), 1134.

${ }^{34}$ Richard Peet, Unholy Trinity: The IMF, World Bank and WTO, 2nd ed. (London: Zed Book, 2003).
}

(c) 2019 Franz Giuseppe F. Cortez

https://www.kritike.org/journal/special issue/cortez april2019.pdf

ISSN 1908-7330

(c) BY-NC-ND 
means privatize, open up, liberalize, offer more incentives to private foreign investment. In short: inject Reaganomics into the Third World." 35

Bagama't naramdaman agad sa ekonomiya ng Pilipinas ang bagsik ng SAP, para sa maraming Pilipino, hindi neoliberalismo kundi si Marcos mismo ang tunay na dahilan ng krisis ng bansa. ${ }^{36}$ Ipinapalagay ni Walden Bello na maraming mga salik sa naging patuloy na pamamayagpag ng ideolohiyang neoliberal noong panahon ni Pangulong Corazon Aquino: pagtatalaga sa pamahalaan ng mga teknokrat at ekonomistang neoliberal, mapait na karanasan sa crony capitalism, kawalan ng alternatibo sa neoliberalismo, pagbagsak ng Sosyalismo sa gitnang Europa, krisis ng sosyodemokratikong estado ng Sweden, ang tila matagumpay na ekonomiya ng Amerika at Gran Britanya dulot ng mga pagbabagong sinimulan nina Reagan at Thatcher, at ang pagpailanglang ng mga bagong industriyalisadong bansa sa Silangang Asya. ${ }^{37}$ Noong panahon ni Pangulong Fidel Ramos, mas lalong naging maimpluwensiya at sumidhi ang pananampalataya sa neoliberalismo. ${ }^{38}$ Ang mga sumunod pang administrasyon mula kay Pangulong Joseph Estrada hanggang kay Pangulong Rodrigo Duterte ay pagpapatuloy lamang ng mga polisiyang nakaugat sa doktrinang neoliberal. ${ }^{39}$

Sa pangkalahatan, ipinapalagay na mas malaki ang naging masamang epekto ng eksperimentong neoliberal sa ating bansa. Lalong lumala ang kahirapan lalong-lalo na ng mga nasa laylayan, samantalang lalo lamang yumaman ang iilan. ${ }^{40}$ Sangkot ang polisiyang neoliberal sa malawakang kawalan ng disenteng hanapbuhay, pagkasira ng kalikasan at di-makatarungang bahagihan ng yaman ng lipunan. ${ }^{41}$

${ }^{35}$ Robin Broad and John Cavanagh, Preface to Unequal Alliance: The World Bank, the International Monetary Fund, and the Philippines (Berkeley: University of California Press, 1988), xvii.

36 Walden Bello, "Neoliberalism as Hegemonic Ideology in the Philippines: Rise, Apogee, and Crisis," in Focus on the Global South (18 October 2009), $<$ https://focusweb.org/neoliberalism-as-hegemonic-ideology-in-the-philippines-rise-apogeeand-crisis/>.

37 Ibid.

38 Walden Bello, "EDSA, Neoliberalism, and Globalization" in Walden Bello (23 March 2017), < $<$ https://www.waldenbello.org/edsa-neoliberalism-and-globalization/>.

39 "Rehashed neoliberal policies: One year of Dutertenomics," in Ibon (28 June 2017), $<$ https://www.ibon.org/rehashed-neoliberal-policies-one-year-of-dutertenomics/>.

${ }^{40}$ Ligaya Lindio-McGovern, "Neoliberal Globalization in the Philippines: Its Impact on Filipino Women and Their Forms of Resistance," Journal of Developing Societies, 23:1-2 (2007).

${ }^{41}$ Benjie Oliveros, “Neoliberalism and greater Inequality," Bulatlat: Journalism for the people (9 June 2016), <https://www.bulatlat.com/2016/06/09/neoliberalism-and-greaterinequality/>. 


\section{Ang SM (Shoe Mart)}

Walang duda na ang pinanggalingan ng salitang SMisasyon ay ang $\mathrm{SM}$, isang negosyo na naging penomenal sa bansang Pilipinas. Halos lahat na yata ng Pilipino ay alam kung ano ang SM. Habang ginagawa ko ang sanaysay na ito, hindi mabibilang kung ilang beses na tumigil ako sapagkat kailangan kong samahan ang aking asawa at anak sa SM. Ang SM ay bahagi na ng buhay Pinoy. Pinoy na Pinoy ang SM. At SM na SM ang Pinoy.

Hindi rin lingid sa maraming Pinoy na ang kamamatay lamang na si Henry Sy, Sr. ang may-ari ng SM, na nagsimula bilang isang maliit na tindahan ng sapatos sa Quiapo, Maynila noong Oktubre 1958. Mula sa isang pangkaraniwang tindahan ng sapatos, nagtayo si Sy ng department stores sa Quiapo, Cubao, at Makati noong dekada '70. Sa gitna raw ng mga agam-agam at pagtutol ng maraming malapit kay Sy, buong tapang na binuksan niya sa publiko ang kanyang unang mall noong 1985 sa North EDSA, Quezon City. Bagama't hindi naman siya ang nagtayo ng unang mall sa Pilipinas, ${ }^{42}$ walang duda na noong nabubuhay pa si Sy, itinuturing siyang hari ng mall at bathala ng mga mallers sa ating bansa. Pinangarap nga raw niya na magkaroon ng branch ng SM "kada-30 hanggang 45 minutong pagmamaneho sa Metro Manila." ${ }^{33}$ Noong 2015, pangako naman ni Hans Sy, isa sa mga anak ni Henry Sy at pangulo ng SM Prime Holdings, Inc., na magiging 75 na ang mga SM malls pagdating ng taong 2018.44 Hindi nambubuladas ang batang Sy sapagkat sa pagtatapos ng taong 2016, 60 na ang SM malls sa bansa, bukod pa sa 6 na SM malls sa Tsina. ${ }^{45}$ Sa pagtatapos naman ng 2017, 7 pang malls sa Pilipinas ang magbubukas. ${ }^{46}$ Batay sa ulat ng online news outlet na Rappler noong 19 Enero 2019, ang SM ay meron nang halos 1,000 retail stores at 77 malls.

42 Ipinangalan sa boksingerong si Muhammad Ali ang Ali Mall sa Araneta Center, Cubao na binuksan noong 1976. Ito ang itinuturing na kauna-unahang mall sa Pilipinas. Kung mas malayang pakahulugan ng mall, babalik tayo sa panahon ng mga Kastila noong 1877 sa Iloilo City kung saan itinayo ng Ingles na si Henry Hoskyns ang unang department store, ang Hoskyns Department Store. Hindi pwedeng tumawad at nagtitinda raw ito ng lahat mula karayom hanggang angkla. Constantino Tejero, "Iloilo: Discover its history, decode its modernity," in Inquirer Lifestyle (11 February 2017), < https://lifestyle.inquirer.net/254095/iloilo-discover-historydecode-modernity/>.

${ }^{43}$ Rolando Tolentino, "Syudad ng malls, Kulturang Popular," in Rolando Tolentino (21 April 2009), <https://rolandotolentino.wordpress.com/2009/04/21/syudad-ng-malls-kulturangpopular-kultura-column-bulatlatcom/>.

${ }^{44}$ Iris Gonzales, "SM Prime eyes 75 malls by 2018," in Philippine Star (2 September 2015), <https://www.philstar.com/business/2015/09/02/1495238/sm-prime-eyes-75-malls-2018>.

45 "SM Prime to open 7 new malls in 2017," in Rappler (30 August 2016), $<$ https://www.rappler.com/business/industries/175-real-estate/144625-sm-prime-new-malls$\underline{2017}>$.

${ }^{46} \mathrm{Ibid}$.

(c) 2019 Franz Giuseppe F. Cortez

https://www.kritike.org/journal/special issue/cortez april2019.pdf

ISSN 1908-7330

(c) $\mathrm{BY}-\mathrm{NC}-\mathrm{ND}$ 
Bukod pa rito, walang duda rin ang galing sa pagnenegosyo ni Sy at ng kanyang mga kasama nang lumawak na ang negosyo mula retailing papunta sa property (mall and office) development, banking, real estate, mga negosyong may kaugnayan sa turismo, at marami pang iba. Ngayon, ang negosyo na nagsimula bilang isang maliit na tindahan ng sapatos sa Quiapo ay ang pinaka-malaki nang business conglomerate sa Pilipinas. ${ }^{47}$ Sampung taon ding itinala ng Forbes si Henry Sy, Sr. bilang pinaka-mayamang Pilipino at naputol lamang ito dahil sa kanyang pagpanaw kamakailan lamang. ${ }^{48}$ Inilarawan ng Forbes na self-made o sariling-sikap si Sy at diversified ang pinanggagalingan ng kanyang kayamanan. ${ }^{49}$

\section{Ano ang SMisasyon?}

Ang ibig kong sabihin sa SMisasyon ng lipunang Pinoy ay ang pagpapatuloy ng proseso ng neoliberalisasyon sa Pilipinas at ang paglawak at pag-igting ng epekto nito sa halos lahat ng aspekto ng buhay ng mga Pilipino: pang-ekonomiya, panlipunan, panrelihiyon, pampulitika, kultural, sikolohikal, moral, ekolohikal, at marami pang iba. Isinasagisag ng SM ang matagumpay at epektibong pagpasok ng neoliberalismo sa bansa.

Hindi ko sinasabi na si Henry Sy ang may pakana ng SMisasyon. Nanagana lamang siya sa bunga ng SMisasyon. Kung nasanay na tayo sa kasabihang "Time is gold," sa kalagayan ni Sy totoong-totoo rin ang "Timing is gold." At dahil siya mismo ang isa sa may pinakamalaking pakinabang, ang buong prosesong ito ay maaaring ipangalan sa mismong negosyo na kanyang itinatag.

Kaugnay ng nabanggit, mahalaga ring bigyang diin na sa proseso ng SMisasyon, hindi lamang SM ang aking pinatutungkulan. Tulad ng sinasabi ni Ritzer ukol sa McDonaldization at ni Bryman sa Disneyization na hindi lamang ito tumutukoy sa McDonald's at sa Disney Company, ${ }^{50}$ sinasabi ko naman ngayon na ang SM ay kumakatawan at sumisimbolo lamang sa mga prosesong may kaugnayan nga sa neoliberalisasyon.

Maraming katangian itong SMisasyon at maraming penomenon ang maaaring pag-aralan na may kaugnayan sa mga praktis ng SM at iba pang malalaking korporasyon sa Pilipinas. Subalit sa pag-aaral na ito, babanggit

\footnotetext{
${ }^{47}$ Chrissee Dela Paz, “Fast Facts: SM Investments Corporation," Rappler (28 June 2017), $<$ https://www.rappler.com/business/170638-fast-facts-sm-investments-corporation-henry-symalls $>$.

48 "Henry Sy tops richest Filipinos list for 10th year in a row - Forbes," in CNN Philippines (25 August 2017), <http://nine.cnnphilippines.com/business/2017/08/24/Forbesriches-Filipinos.html $>$.

49 "Henry Sy," in "2018 Philippines 50 Richest Net Worth," in Forbes (as of 5 September 2018), <https://www.forbes.com/profile/henry-sy/?list=philippines-billionaires\#2cec94e14a10>.

${ }^{50}$ Bryman, "The Disneyization of Society," 160.
}

(C) 2019 Franz Giuseppe F. Cortez https://www.kritike.org/journal/special issue/cortez april2019.pdf ISSN 1908-7330 
lamang ako ng tatlong penomenon na masasabi kong bahagi ng proseso ng SMisasyon: (1) walang-patumanggang pagkonsumo, (2) kultura ng matiraang-matibay, at (3) mito ng sipag at tiyaga. ${ }^{51}$ Ipinapakita nito na ang neoliberalismo ay hindi lamang ekonomikong kalabisan ng Kapitalismo. Isa rin itong ideolohiya at anyo ng pamamahala na sabay nagpapalakas at nagpapatibay ng mga pagpapahalagang pinanghahawakan ng pinagpanibagong mukha ng Kapitalismo.

\section{Walang-Patumanggang Pag-Konsumo (Hyper-Consumerism)}

Ang isang ibig kong sabihin sa SMisasyon ay ang pag-igting ng kultura ng konsumerismo sa Pilipinas. Noong 1998, sinabi ni Steven Miles na sa mga huling taon ng ika-20 siglo, konsumerismo na nga ang bagong relihiyon habang pinapasok na nito ang ating pang-araw-araw na buhay. ${ }^{52}$ Mga ilang taon bago pa nito, inilarawan ni Brian Appleyard ang konsumerismo bilang isang relihiyon: ang mga naglalakihang mall ang mga katedral at ang pag-konsumo ng mga produkto at serbisyo ang anyo ng pagsamba. ${ }^{53}$ Binanggit nina Rico at De Leon na isinasagisag ng pagdami ng mga mall sa Pilipinas ang kultura ng konsumerismo. ${ }^{54}$ Ayon kay Tolentino, "galing sa global na istruktura ng konsumerismo ang malling, at kaiba lang ang adaptasyon ng negosyante at konsumeristang Filipino sa istrukturang ito." 55

Magkaiba ang consumption sa consumerism. Ang consumption ay tumutukoy lamang sa payak na pagbili at paggamit ng produkto, isang awtomatikong gawain na para kay Zygmunt Bauman ay maihahanay sa mga

51 Tinanong ng reviewer ng papel na ito kung bakit hindi nabanggit ang usapin ng "kontraktwalisasyon, pambabarat sa mga kawani, union busting, panggagago sa mga kababaihan sa pamamagitan ng pagsusuot ng mga high heels at make up at maikling palda." Naisip ko na rin na bahagi ng SMisasyon ang mga penomenang ito. Subalit hindi sapat ang espasyo upang talakayin ang mga ito. Pinaplano ko na itong maging bahagi ng pagpapatuloy ng pag-aaral ukol sa SMisasyon. O kaya naman, baka merong mambabasa na mahikayat na pag-aralan ang iba pang mukha ng SMisasyon ng lipunang Pinoy. Ang aking papel (paper) ay isang paanyaya. At ang aking papel (role) din ay pumukaw ng kaisipan.

52 Steven Miles, Consumerism as a Way of Life (London: Sage, 1998), 1.

${ }^{53}$ Brian Appleyard, "Shopping around for salvation: The new religion is consumerism and massive malls are its cathedrals. Let us bow our heads and pay," in The Independent (3 November 1993), < https://www.independent.co.uk/voices/shopping-around-for-salvation-thenew-religion-is-consumerism-and-massive-malls-are-its-cathedrals-1501792.html>.

${ }^{54}$ Jore-Annie Rico and Kim Robert C. de Leon, "Mall culture and consumerism in the Philippines," in State of Power 2017, ed. by Nick Buxton and Deborah Eade (Amsterdam, Netherlands: Transnational Institute, 2017), 1-9, <https://www.tni.org/files/publicationdownloads/stateofpower2017-mall-culture.pdf>.

${ }^{55}$ Rolando B. Tolentino, "Mall, malling, nagmo-malling...," in Pinoy Weekly (23 January 2010), <https://www.pinoyweekly.org/2010/01/mall-malling-nagmo-malling\%e2\%80\%a6/>.

(c) 2019 Franz Giuseppe F. Cortez

https://www.kritike.org/journal/special issue/cortez april2019.pdf

ISSN 1908-7330

(c) BY-NC-ND 
biyolohikal na proseso na kailangang gawin upang mabuhay. ${ }^{56}$ Samantalang ang consumerism ay tumutukoy sa mas kumplikadong paraan ng pamumuhay na sobra-sobrang nakatuon sa pagbili at paggamit ng produkto. ${ }^{57}$ Sinabi pa ni Bauman na kung ang consumption ay katangian ng isang indibidwal na tao, ang konsumerismo ay katangian ng lipunan. 58

Sa kasalukuyang anyo ng lipunan na pinapangisawaan ng mga polisiyang nakabatay sa pinagpanibagong Kapitalismo, ang kultura ng konsumerismo ay higit pang bumabaon sa kaibuturan ng pagkatao ng mga kasapi nito. Dahil maraming produksyon, kailangang maraming bumili ng mga produkto. Dahil hindi titigil ang paggawa, kailangang hindi rin tumigil ang pag-ubos ng mga ginawa. Isang proseso na parang walang katapusan. Isang proseso na kakambal na ng Kapitalismo mismo. ${ }^{59}$

Ang pag-igting ng kultura ng konsumerismo ay masasabing bahagi ng proseso ng SMisasyon sapagkat malaki ang nagiging papel ng naglalakihang mall sa Pilipinas sa prosesong ito. ${ }^{60}$ Kabilang ang mall sa tinatawag ni Ritzer na sites of consumption. ${ }^{61}$ Oo nga't hindi naman ang penomenon ng mall at malling ang ugat ng konsumerismo. Oo nga't bago pa ipinatayo ang unang mall sa Pilipinas, nagsimula na ang paglaganap ng kulturang ito. Oo nga't masasabi rin na ang konsumerismo ay nangyayari sa pagsasanib ng iba't ibang pwersa na makikita sa loob at labas ng mall. Ang mga institusyon katulad ng pamilya, paaralan, media, negosyo, at pamahalaan ay sangkot sa pag-igting ng konsumerismo.

Subalit hindi rin maipagkakaila na ang malling phenomenon na pangunahing kinakatawan ng mga SM mall ay may malaking ambag sa pagigting ng kultura ng konsumerismo sa maraming Pilipino. Sa loob ng isang mall, kalimitang isinasakatuparan ang konsumeristikong nasà (consumeristic desire) sa pamamagitan ng pagbili at pagkonsumo ng mga produkto at serbisyo. Naka-disenyo ang mga mall upang maghalo at maglaho ang mga hangganan ng pangangailangan at pagnanasa. Ang ideya ng window shopping ay isang mapanuksyong eksena upang bumili. Ang kabi-kabilang sale at eat-

${ }^{56}$ Zygmunt Bauman, Consuming Life (Cambridge: Polity, 2007), 25.

57 "[C]onsumerism is the cultural expression and manifestation of the apparently ubiquitous act of consumption." Miles, Consumerism as a Way of Life, 4.

${ }^{58}$ Bauman, Consuming Life, 29.

${ }^{59}$ Chris Arthur, Financial Literacy Education: Neoliberalism, the Consumer and the Citizen (Rotterdam: Sense Publishers, 2012).

60 "...those places in society where commodities are most dramatically present in our lives the shopping mall, the showroom, the advertisement-have become sites of cultural production in which economic goods are transformed into components of complex meaning systems, reversing the evolutionary separation of culture and economy." Robert Dunn, Identifying Consumption: Subjects and Objects in Consumer Society (Philadelphia: Temple University Press, 2008), 64.

61 George Ritzer, Douglas Goodman, and Wendy Wiedenhoft, "Theories of Consumption," in Handbook of Social Theory, ed. by George Ritzer and Barry Smart (London: Sage, 2001), 421. 
all-you-can ay isang panunukso upang bumili nang bumili kahit hindi naman kailangan at kumain nang kumain kahit busog naman. Ang mga makukulit at mapanlitong istratehiya ng pagsasabi sa mamimili na magmadali kung hindi ay mauubusan ay katulad ng sinasabi rin ni Stephen Bertman na hurried culture at nowist culture na kaugnay na rin ng kultura ng konsumerismo. ${ }^{62}$ Pinadadali at pinadudulas din ang proseso ng paggastos sa pamamagitan ng paglalagay ng mga ATM at bangko sa loob ng mga mall. Ang pagkasalat sa pera ay panandaliang nilulutas ng mga credit card at iba't ibang programa ng pagpapautang. Ang pagkasawa sa produkto-mapa-sapatos, damit, cellphone, o telebisyon man - ay nalulunasan sa pamamagitan ng patuloy at mabilisang pagbabago ng mga display ${ }^{63} \mathrm{o}$ planado at inaakalang pagkalaos ng mga produkto. ${ }^{64}$ Sa aliw ng mga ilaw, halina ng mga ingay, laro ng mga kulay, maginhawang karanasan, at larawan ng mga masasayang modelo at naggagandahang artista, nahahalina ang flâneur na bumili nang bumili, kumain nang kumain, gumastos nang gumastos kahit na sa mga bagay na hindi naman talaga tunay na kailangan. Sa pamamagitan ng magulong pagkakaayos (o maayos na pagkakagulo?) ng SM, nalilito at nabubulagan ang mamimili sa kanilang pagpili kung ano ang pagkakaiba ng pangangailangan sa pagnanasa lamang. Kaya nga, pag masaya nang nabili ang isang bagay, uuwi at mapagtatanto na hindi naman pala talaga kailangan ang mga ito. Masayáng pagsasayáng.

Kung sinasabi ni Bryman na sa Disneyization, ang mamimili ang soberenya, sa SMisasyon, kunwari lamang ang kalayaang pumili ng mamimili. Sa sinasabi kong SMisasyon, ang mamimili (consumer/buyer) ay hindi mamimilì (one who chooses or makes a choice). Totoong-totoo ang sinasabi ni Jean Baudrillard na kunwari lamang ang rasyonalidad at kalayaan ng tao sa proseso ng konsumerismo; lahat ay napapailalim sa kapangyarihan ng mga tanda (sign) at imahe (image). ${ }^{65}$

Dahil sa iba't ibang mga taktikang nabanggit, mas makatwirang tanungin kung meron ba talagang soberenya at kalayaan ang mamimili. Sa set-up ng mall, mukhang wala talaga. Sa pananaw ni Tolentino:

\footnotetext{
${ }^{62}$ Stephen Bertman, Hyperculture: Human Cost of Speed (Westport: Praeger, 1998).

${ }^{63}$ Michael Billig, "Commodity Fetishism and Repression: Reflections on Marx, Freud and the Psychology of Consumer Capitalism," Theory and Psychology, 9:3 (1999), 325.

64 "...the advent of consumerism augurs the era of inbuilt obsolescence of goods offered on the market and signals a spectacular rise in the waste-disposal industry..." Bauman, Consuming Life, 31. Tungkol sa usapin naman ng planned and perceived obsolescence, tingnan: Tim Hindle, The Economist Guide to Management Ideas and Gurus (London: Profile Books, 2008), 147-148; Giles Slade, Made to Break: Technology and Obsolescence in America (USA: Harvard University Press, 2006); Joseph Guiltinan, "Creative Destruction and Destructive Creations: Environmental Ethics and Planned Obsolescence," Journal of Business Ethics, 89 (2009), 19-28.

65 Jean Baudrillard, The Consumer Society: Myths and Structures (London: Sage Publications, 1998), 79-80.
}

(c) 2019 Franz Giuseppe F. Cortez https://www.kritike.org/journal/special issue/cortez april2019.pdf ISSN 1908-7330

(cc) BY-NC-ND 
Iniiwan ng maller ang kaniyang moda ng produksyon ng paggawa, para gawing purong sityo ng libangan ang mall. Hindi niya maalala kung paano siyang nakarating dito-kung paanong nagpursigi para kumita, ginamit ang lakas-paggawa, ibinenta ito sa eksplotatibong relasyon-pero masaya siya sa pagdating dito. ${ }^{66}$

Hindi nakapagtataka ang pagkalimot na ito na sinasabi ni Tolentino sapagkat sinadya at pinag-isipan ang lahat-lahat nitong sangkap upang ang tao ay bumili at magwaldas. Ang mga unang mall na parang mga kahon ng sapatos ay naghihiwalay ng labas at loob at nagpapalabo ng kanina, ngayon, at mamaya.

Mabuti ring tingnan ang ugnayan ng hyper-consumerism at ang sinasabi ni Karl Marx na commodity fetishism. Kung may halaga man si Marx para sa atin ngayon, itinuturing ko na ang konsepto ng commodity fetishism ang isa sa mga halagang ito. Sa isang maikling bahagi ng Das Kapital, ipinaliwanag ni Marx ang fetishism of commodity. Sa aking payak na pagunawa, sinasabi ni Marx na kapag ang isang bagay, tulad ng mesa, ay naging isang produkto na dinala sa pamilihan (commodity), nakakalimutan na ang iba't ibang ugnayang panlipunan (social relations) na pinagdaanan ng nasabing produkto: ang pumutol ng kahoy, ang gumawa ng mesa, ang naghatid sa pamilihan, at iba pa. Sa halip, napapalitan ito ng ugnayan sa pagitan ng mga bagay: pera at produkto ;67 para bang nagkakaroon ng kakaibang kapangyarihan ang produkto: anting-anting, agimat, fetish.

Ngayon, sa loob ng mall, tuluyan na ngang natabunan ang mga ugnayang panlipunan na ito. Hindi na maisasaisip ng bumibili ng sapatos o damit o prutas o cellphone o diamond ring, ang mga manggagawang nagbuhos ng dugo, pawis, at luha upang mabuo ang mga produktong ito. Hindi na mamamalayan ng mga mamimili kung meron bang karahasan, kawalangkatarungan, at pagsagasa sa mga karapatang pantao habang hinuhukay, itinatanim, ginagawa, at inililipat ang mga nasabing produkto. ${ }^{68}$ Sa isang kultura ng hyper-consumerism, naglaho na ang makataong karakter ng produkto. Sa halip, nagiging purong bagay na nga lamang ito na may katumbas na halagang pera. ${ }^{69}$

\footnotetext{
${ }^{66}$ Rolando Tolentino “Kabataang Katawan, Mall at Siyudad: Mga Tala sa Gitnang Uring Karanasan at Neoliberalismo" Philippine Humanities Review, 14:12 (April 2012), 70.

${ }^{67}$ Tingnan sa Karl Marx, "The Fetishism of the Commodity and Its Secret," in Capital: A Critique of Political Economy, Vol. 1, trans. by Ben Fowkes (Middlesex, England: Penguin Books, Ltd., 1976), 163-177.

${ }^{68}$ Jones, ten Bos, and Parker, For Business Ethics, 103-105.

${ }^{69}$ Billig, "Commodity Fetishism and Repression," 315-316.
} 


\section{SMISASYON NG LIPUNAN}

Makatutulong din ang pagsusuri ni Baudrillard tungkol sa consumer society. Malawak ang kanyang pag-aaral kaya hihiramin ko lang ang kanyang konsepto ng profusion na isasalin ko bilang "kasandamakmakan."70 Sa obserbasyon ni Baudrillard, ang gabunton at sandamakmak na mga produkto sa mga Parisian department store at drugstore ${ }^{71}$ ay nagpapakita na labis-labis ang mga produkto para sa lahat at na nalutas na ang problema ng kakulangan. Paano nga namang masasabing may kakulangan kung nakabuyangyang ang kasandamakmakan?

There is something more in this piling high than the quantity of products: the manifest presence of surplus, the magical, definitive negation of scarcity... Our markets, major shopping thoroughfares and superstores also mimic a new-found nature of prodigious fecundity. These are our Valleys of Canaan where, in place of milk and honey, streams of neon flow down over ketchup and plastic. ${ }^{72}$

Pinansin din ni Baudrillard kung paanong nagawang pagsama-samahin sa loob ng Parisian drugstore ang halos lahat ng mga gawain ng tao.

Work, leisure, nature and culture: all these things which were once dispersed, which once generated anxiety and complexity in real life, in our 'anarchic and archaic towns and cities,' all these sundered activities, these activities which were more or less irreducible one to another, are now at last mixed and blended, climatized and homogenized in the same sweeping vista of perpetual shopping. ${ }^{73}$

Makatwiran at angkop nga marahil na tawaging "city" ang mga mall na ginagawa ng SM. Sa isang banda, maihahanay sila sa Quezon City, Cebu City, Davao City, Batangas City, at iba pa. Ang mall ay isang city within a city. Halos lahat na nga yata ng pang-araw-araw na gawain ng tao ay maaari na niyang

70 Ang "sandamakmak" ay isang palasak na pang-uring nangangahulugan ng pagiging "sobra-sobra" o "labis-labis." Kahalintulad ito ng mga palasak ding salitang "sangkaterba," "sanlaksa," at "sandamukal." Halimbawa, "Sandamakmak na basura ang nakuha sa Manila Bay." Tingnan ang Michael L. Tan, "'Sandamakmak'," in Inquirer Opinion (15 August 2018), <https://opinion.inquirer.net/115379/sandamakmak>. Ang "kasandamakmakan" ay ang pangngalan ng "sandamakmak."

${ }^{71}$ Hindi dapat malígaw ang mga mambabasa. Sa konteksto ni Baudrillard, ang drugstore sa Paris ay hindi lamang nagbebenta ng gamot. Kahalintulad ito ng mga makabagong shopping centers.

72 Baudrillard, The Consumer Society, 26.

${ }^{73}$ Ibid., 30.

(c) 2019 Franz Giuseppe F. Cortez

https://www.kritike.org/journal/special issue/cortez april2019.pdf

ISSN 1908-7330

(c) BY-NC-ND 
isagawa rito. Bumili ng sapatos, sipilyo, at sinulid. Mamalengke at kumain sa eat-all-you-can. Manalangin sa Diyos, manood ng sine, at maka-meet-and-greet ang paboritong artista. Magpasuso ng sanggol, magpa-alaga ng musmos at magpa-tutor ng anak. Pumasok sa museo at massage sauna. Magbayad ng kuryente at mag-deposito sa bangko. Magpagawa ng relo at magpa-xerox ng biodata. Magbayad ng buwis, mag-renew ng lisensya, at bumoto. Magpatingin sa doktor at kumanta sa videoke. Ad infinitum. Sabi nga ng tagline ng SM: We've got it all for you! Sandamakmak ang makikita at maaaring gawin sa SM. Profusion. Kasandamakmakan ng produkto, serbisyo, at ng kung ano pang maaaring ikonsumo.

Hindi maralita ang Pilipinas. At hindi naghihirap ang mga Pilipino. Maayos ang lipunan. Sagana ang produkto. Umaapaw ang yaman. Sobrasobra para sa lahat. Ito ang imahe ng kasandamakdamakan na nais ipahiwatig at iparanas sa loob ng mall. Ito ang pangako ng neoliberalismo: kalayaang pumili mula sa kasandamakmakan. Pwedeng tumingin, humawak, at umamoy. May produktong naaangkop sa bawat isa. At kung hindi mo kayang bilhin, wala sa kasaganaan ang problema. Sapagkat paanong magiging mali ang isang sistemang nakalilikha ng kasandamakmakan? Paanong magiging mali ang kasandamakmakan na sabay na nagbibigay ng kalayaang pumili? ${ }^{74}$ Kung may mali man, naroon ito sa indibidwal na hindi marunong maghanda ng sarili at bumagay sa kontemporanyong balangkas ng ekonomiya at lipunan. ${ }^{75}$

\section{Matira ang Matibay (Survival of the Fittest)}

Ang ibig ko pang sabihin sa SMisasyon ng lipunang Pinoy ay ang pagpapalaganap at pagpapatibay ng paniniwala na ang larangan ng negosyo at buhay ekonomiko ay maihahalintulad sa isang gubat na kung saan matira ang matibay, isang mabalasik at madugong paligsahan. Itong ganitong uri ng pananaw ay karaniwang iniuugat sa isang anyo ng Social Darwinism na pinangangalandakan ni William Graham Sumner, isang Amerikanong antropolohista. Para kay Sumner, upang magkaroon ng pag-unlad sa isang lipunan, kailangan talaga na makipagbuno ang mga matitibay at sukdulang masagasaan ang mga mahihina. ${ }^{76}$ Ang batas daw ng biological evolution $\mathrm{ni}$ Charles Darwin ay totoo at angkop din sa pag-unlad ng mga institusyon ng lipunan, kasama na ang komersyo.

${ }^{74}$ Eva-Lotta Hedman and John Sidel, Philippine Politics and Society in the Twentieth Century: Colonial Legacies, Postcolonial Trajectories (London: Routledge, 2000), 134.

${ }^{75}$ Amirhosein Khandizaji, Baudrillard and the Culture Industry: Returning to the First Generation of the Frankfurt School (Switzerland: Springer Nature, 2017), 103.

${ }^{76}$ David Lea, "Darwinism and Ethics," in Encyclopedia of Business Ethics, ed. by Robert Kolb (Los Angeles: Sage Publications, 2008), 545.

(C) 2019 Franz Giuseppe F. Cortez https://www.kritike.org/journal/special issue/cortez april2019.pdf ISSN 1908-7330 


\section{SMISASYON NG LIPUNAN}

Gamit ang kaisipan ni Sumner, ikinatwiran ng mga kapitalista noong mga huling taon ng ika-19 na siglo na ang mga milyonaryong burgis tulad nina John D. Rockefeller at Andrew Carnegie ang modelo ng pag-unlad ng ebolusyon ng lipunan. Sila ang matitibay at angkop na magpatuloy sa paggulong ng pag-unlad ng lipunan. Sila ang nanatiling nakatayo sa paligsahan ng merkado. Gamit ang kaisipan ni Adam Smith tungkol sa "invisible hand" at ang pag-aaral ni Charles Darwin ukol sa biological evolution, sinabi ng mga taga-suporta ng ganitong anyo ng Social Darwinism na kahit na sa larangan ng pagnenegosyo, merong batas ng kalikasan na matitira at magtatagumpay ang matitibay at malalakas, samantalang titiklop naman at maglalaho ang mga mahihina at mga walang kakayahang sumabay sa mga pagbabago.

Sa panahon ng neoliberalismo, lalong umigting ang paniniwala sa prinsipyo ng "matira ang matibay." Kahit si Pope Francis ay ganito ang hinaing:

Today everything comes under the laws of competition and the survival of the fittest, where the powerful feed upon the powerless. As a consequence, masses of people find themselves excluded and marginalized: without work, without possibilities, without any means of escape. ${ }^{77}$

Ipinaliwanag naman ni Pierre Bourdieu na ang Darwinian world na pinalakas muli ng neoliberalismo ay nagpapaigting din ng kumpetisyon hindi lamang sa pagitan ng mga korporasyon kundi sa lebel din ng mga indibidwal sa loob mismo ng isang organisasyon. ${ }^{78}$ Sa pananaw naman ni Harvey, ipinapalagay ng neoliberalismo na ang kumpetisyon sa pagitan ng bawat indibidwal, ng bawat organisasyon, ng bawat teritoryo ay isang pangunahing kagalingan (virtue) ng lipunan. ${ }^{79}$

Sa Estados Unidos, merong tinatawag na penomenon ng Walmartization. Tumutukoy ito sa pagdating ng isang higanteng negosyong sumisira sa maliliit na negosyo at kung kaya't ang mga nawalan ng trabaho ay napipilitang tumanggap ng mga trabaho sa dumating na higanteng negosyo sa kabila ng mas maliit na sweldo. ${ }^{80}$

77 Francis, Evangelii Gaudium, Apostolic Exhortation (24 November 2013), § 53, $<$ http://w2.vatican.va/content/francesco/en/apost exhortations/documents/papafrancesco_esortazione-ap_20131124_evangelii-gaudium.html>.

78 Pierre Bourdieu, Acts of Resistance: Against the Tyranny of the Market, trans. by Richard Nice (New York: The New Press, 1998), 97.

${ }^{79}$ Harvey, A Brief History of Neoliberalism, 65.

${ }^{80}$ Sariling salin ng depenisyon ng "Walmartization" mula sa New Word Suggestion ng Collins Dictionary: "When a large chain store moves into a region and devastates local businesses driving displaced workers into low paying chain store jobs."

(c) 2019 Franz Giuseppe F. Cortez https://www.kritike.org/journal/special issue/cortez april2019.pdf ISSN 1908-7330

(c) BY-NC-ND 
Hindi naman mahirap makita ang pilosopiya ng matira-ang-matibay sa mga pagkilos ng SM. Katulad ng proseso ng Walmartization, ang SMisasyon din ay isang proseso na kung saan ang mga lokal na negosyante ay unti-unting sumusuko sa tibay, lakas, at kapangyarihan ng malalaking negosyante katulad ng SM. Kasabay ng tuwa at galak ng mga tao sa pagdating ng SM sa kanilang lugar ay ang panlulumo naman ng maraming maliliit na negosyo na nanganganib at mapipilitang magsara kung hindi maghahanda at magiging mas entrepreneurial pa lalo. Nilalamon ng higanteng negosyong ito ang maliliit na negosyo sa gilid-gilid. Oo nga't lumilikha ito ng trabaho, nag-aambag sa buwis ng mga lokal na pamahalaan, at maaaring lumikha rin ng iba pang maliliit na negosyo, ngunit hindi rin maipagkakaila na maraming nasasagasaan sa pagpasok ng SM. Kahit na nga ang simpleng tindera ng turon ay nanganganib sa turon ni Herbert Sy (vice chairman ng SM Markets) na nagpasok ng halagang PhP 360 milyon (24 milyong piraso ng turon) para lamang sa taong 2016. ${ }^{81}$ Ang tindahan ni Aling Nena ay titiklop sa SaveMore, HyperMart, at AlphaMart-mga maliliit (pero malalaki rin) na retail store na pag-aari rin ng konglomerasyon ng SM. Katulad ng jeepney na "hari ng kalsada," marahil nanganganib ding maglaho ang sarisari store na maituturing namang "reyna ng kanto."

Ang paligsahang ito kung saan sigurado na kung sino ang mananalo ay hindi lamang nangyayari sa pagitan ng higanteng SM at mga bulilit na negosyante sa isang lokal na pamayanan. Mismong sa loob ng SM, nararamdaman ng mga nangungupahan kung paanong nakikipagpaligsahan mismo sa kanila ang panginoong may-mall. Kung merong tindang lechon ang Mila's, nagtitinda na rin ng lechon ang SM mismo. Kahit ang konsepto ng SM Bonus ay maaaring tingnan bilang isang anyo ng pakikipagpaligsahan ng SM sa mismong mga nangungupahan sa kanya.

Subalit sa pananaw ng neoliberalismo, ito nga raw ang punto ng kumpetisyon sa merkado: matira ang matibay-batas ng gubat, batas ng pamilihan, batas ng kalíkasan ng tao at hayop. Hindi naman talaga pwedeng lahat ay mananalo. Merong malalagas at uuwing luhaan. Kahit si Hans Sy mismo kailangang maging alerto sa hamon ng online selling at makinig kay Jack Ma, may-ari ng Alibaba na binili na rin ang malaking bahagi ng Lazada. ${ }^{82}$

Ipinapakita ng penomenon ng SM kung paanong natitira ang matibay at marunong sumabay. Ngunit sabay ring ipinapahiwatig ng SM ang isang kabalintunaan: na ang paligsahan sa merkado ay hindi naman talaga isang patas na paligsahan sapagkat meron laging malakas na tatalo sa mahina, matibay na tatalo sa mabuway, makapangyarihan na tatalo sa

81 "How SM won with turon," in SM Investments Corporation (30 May 2017), $<$ https://www.sminvestments.com/company-releases/how-sm-won-turon>.

${ }^{82}$ Lala Rimando, "Forbes Philippines: Reinventing the mall," in SM Prime (8 April 2016), <https://smprime.com/media/news/forbes-philippines-reinventing-mall>. 
ordinaryo, tuso na tatalo sa matapat. Kahit na iniisip niya ang konteksto ng pandaigdigang merkado, nababagay rito ang puna ni Harvey: "While the virtues of competition are placed up front, the reality is the increasing consolidation of oligopolistic, monopoly, and transnational power within a few centralized multinational corporations..." 83 Hindi na kagulat-gulat kung, isang araw, magigising na lamang tayo na pag-aari na ng SM at ng iba pang malalaking negosyo kahit na ang kasuluk-sulukang bahagi ng Pilipinas. Philippines under siege!

\section{Mito ng Sipag at Tiyaga}

Ang ibig ko pang sabihin ng SMisasyon ng lipunang Pinoy ay ang lumalaganap nang kaisipan at pakiramdam na kapag masipag at matiyaga, meron talagang mapapalang nilaga. Ginamit pa nga itong slogan sa kampanya dati ni Manny Villar na itinuturing na ngayong pinakamayamang Pilipino dahil na rin sa negosyong real estate at mga mall. ${ }^{84}$

Sa kaibuturan ng pananaw na ito, sinasabi na kahit saan ka pa nanggaling, kahit ano pa ang katayuan mo sa buhay, kumpleto man o kulang-kulang ang mga bahagi ng iyong katawan, binibigyan ka ng lipunan ng pag-asa at pagkakataon na umangat at umunlad. Lahat ay may oportunidad na marating ang tugatog ng tagumpay. Patas at patag ang palaruan. Ito ang tinatawag ni Jo Littler na meritocratic feeling. ${ }^{85}$

Sa artikulo ni George Monbiot, sinabi niya na bahagi rin talaga ng ideolohikal na aspeto ng neoliberalismo ang pagpapalaganap ng kaisipan na umuunlad ang buhay ng mga taong nagsisikap at nagsasakripisyo. ${ }^{86}$ Binigyang diin naman ni Simon Clark ang pag-aangat ng kaisipang ito sa lebel ng etika ng merkado: "[t]he market was not just an economic, but also a moral force, penalizing the idle and incompetent and rewarding the enterprising and hardworking." 87 Para kay Littler, sa pangkasalukuyang panahon ng neoliberalismo, ang pangako ng meritokrasya ay mas lalong pinalalakas habang sabay na ipinapamukha na ang kumpetisyon ay isang obligasyong

\footnotetext{
${ }^{83}$ Harvey, A Brief History of Neoliberalism, 80.

${ }^{84}$ Sa kasalukuyan din, naitayô na ang malawakang livelihood program ng mga Villar sa ilalim ng kanilang Villar Sipag at Tiyaga Foundation.

85 Jo Littler, "Meritocracy as Plutocracy: The Marketising of Equality within Neoliberalism," New Formations: A Journal of Culture/Theory/Politics, 80-81 (2013), 52-72.

${ }^{86}$ George Monbiot, "Neoliberalism - the ideology at the root of all our problems," in The Guardian (15 April 2016), <https://www.theguardian.com/books/2016/apr/15/neoliberalismideology-problem-george-monbiot $>$.

${ }^{87}$ Simon Clarke, "The Neoliberal Theory of Society," in Neoliberalism: A Critical Reader, 51.

(c) 2019 Franz Giuseppe F. Cortez https://www.kritike.org/journal/special issue/cortez april2019.pdf ISSN 1908-7330
}

(c) BY-NC-ND 
moral. Kung kaya't hindi nag-atubili si Littler na tawagin itong neoliberal meritocracy. ${ }^{88}$

Bakit ko naman nasabing angkop na isama ang meritokratikong pakiramdam sa penomenon ng SMisasyon? Meron pa nga bang kwento na mas inspiring pa kaysa sa rags-to-riches story nitong si Henry Sy? ${ }^{89}$ Sabi nga ni Salvador Panelo, kinakatawan raw ni Sy ang Pilipinong masipag. ${ }^{90}$ Ang naglalakihang SM mall at condominium ang animo'y mga buhay na larawan na kumikiliti sa imahinasyon ng Pinoy na talagang may pag-asang umunlad, kahit na ang pinaka-abang mamamayan ng Pilipinas. Huwag ka nang magugulat kung makita mo na lang si Sy at mga katulad niya sa ating mga aklat-aralin bilang modelo ng pagiging masikap, masipag, matiyaga, matapang, matalino, malikhain, madiskarte, marunong magsakripisyo para sa pangarap, at lahat na nga siguro ng mga katangian ng isang magaling na negosyante at entrepreneur. Hindi na lamang si Jose Rizal o Ninoy Aquino ang mga bayani na kung saan ang mga pangalan ay nakaukit sa mga gusali ng paaralan. Si Sy na rin ang bagong bayani para sa isang bagong Pilipinas na naghahangad ng ekonomikong kaunlaran. May mga gusali ngang nakapangalan na sa kanya at sa iba pang mga negosyanteng mga simbolo ng bagong mukha ng Kapitalismo. Mga gusaling katas ng sipag, sikap, tiyaga, determinasyon, tapang, paniniwala sa Maykapal, at galing ng mga negosyanteng Pinoy. Para bang sinasabi sa mga makakakita na sila ang dapat tularan. Sila ang mga makabagong halimbawa ng tagumpay. Sila ang mga bagong mukha nina Rizal, Bonifacio, Mabini, at Jacinto.

Subalit tulad ng barya, dalawa ang mukha ng meritokrasya: na kung hindi ka naging matagumpay bagkus ay naging hari ng sablay, malamang kaysa hindi, kulang ka sa sipag at tiyaga, sa sakripisyo at sampalataya, sa tapang at tiwala. Para bang nahawahan ng eksistensyalismo ang ideolohiyang ito: kung ikaw ay sumablay, wala kang ibang dapat sisihin kundi ang sarili. Sa diskurso ng neoliberalismo, nasa bawat indibidwal ang kanyang kapalaran. Kung wala kang trabaho, hindi balangkas ng ekonomiya at ng lipunan ang may problema, kundi ikaw. Hindi mo inihahanda ang iyong sarili sa mga pangangailangan ng sistemang kinapapalooban mo. ${ }^{91}$ Walang mali sa istruktura at sistema; nakatuon sa indibidwal ang sisi at papuri.

${ }^{88}$ Jo Littler, Against Meritocracy: Culture, Power and Myths of Mobility (London: Routledge, 2018), 3 ff.

89 Tingnan ang kanyang kwento sa Hedman and Sidel, Philippine Politics and Society in the Twentieth Century, 130-133.

${ }^{90}$ Aika Rey, "Malacañang: Henry Sy Sr a 'pillar of Philippine economy'," in Rappler (19 January 2019), <https://www.rappler.com/nation/221351-malacanang-statement-henry-sy-srpillar-philippine-economy>.

${ }^{91}$ Julio Cesar de Castro, "The Consumer as Agent in Neoliberalism," Matriz, 9:2 (2015), 278.

(C) 2019 Franz Giuseppe F. Cortez https://www.kritike.org/journal/special issue/cortez april2019.pdf ISSN 1908-7330 


\section{SMISASYON NG LIPUNAN}

Ngunit para sa mga kritiko ng neoliberalismo, hindi naman totoo na patas ang labanan at patag ang palaruan. Sa simula pa lang, doktorado na ang panuntunan at manipulado na ang paligsahan. Sa bawat isang matagumpay na Henry Sy, sangkaterbang masisipag at matitiyaga ang uuwing luhaan, na kung minamalas-malas pa, ay wala na ring mauuwian. ${ }^{92}$ Di-maliparang uwak ang espasyo sa ilalim samantalang parang jeepney sa rush hour ang lugar sa itaas: unahan, gulangan, siksikan, balyahan, bulyawan. ${ }^{93}$

Hindi nabibigyang-pansin ang iba't ibang panlipunan, pampulitika, at pang-ekonomiyang salik, na para bang perpekto at swabe ang mga balangkas ng lipunan. Nakakalimutan ang sinasabi ni Aneel Karnani na malaking pagkakaiba ng materyal, sikolohikal, pang-ekonomiko, at panlipunang reyalidad ng mga mahihirap kung ihahambing sa mga mayayaman. ${ }^{94}$ Ganito rin ang reklamo sa neoliberalismo ni Jeff Sugarman:

There is diminishing appreciation that individuals' predicaments are a product of more than simply their individual choice, and include access to opportunities, how opportunities are made available, the capacity to take advantage of opportunities offered, and a host of factors regarding personal histories and the exigencies of lives. ${ }^{95}$

Kumakampi rin dito si Monbiot nang sinabi niya na:

The rich persuade themselves that they acquired their wealth through merit, ignoring the advantages - such as education, inheritance and class - that may have helped to secure it. The poor begin to blame themselves for their failures, even when they can do little to change their circumstances. ${ }^{96}$

Sa mito ng sipag at tiyaga, totoong totoo nga ang pahayag ni Pierre Bourdieu ng kahalagahan ng kultural na kapital sa pag-unlad ng isang tao sa lipunan. Hindi dumadaloy nang ganun-ganon na lamang ang yaman ng lipunan sa

92 "Neoliberalism is an economic and political program benefiting the top 1 percent of the world's population as it sends billions to poverty." Jodi Dean, "Enjoying Neoliberalism," Cultural Politics, 4:1 (2008), 67.

${ }^{93}$ Tingnan ang James Bloodworth, The Myth of Meritocracy: Why Working-Class Kids Get Working-Class Jobs (London: Biteback Publishing, 2016), 49 ff.

${ }^{94}$ Aneel Karnani, "Romanticizing the Poor," Stanford Social Innovation Review (Winter 2009), 38-43

${ }^{95}$ Jeff Sugarman, "Neoliberalism and Psychological Ethics," in Journal of Theoretical and Philosophical Psychology, 35:2 (1995), 105.

${ }^{96}$ Monbiot, "Neoliberalism - the ideology at the root of all our problems."

(c) 2019 Franz Giuseppe F. Cortez

https://www.kritike.org/journal/special issue/cortez april2019.pdf

ISSN 1908-7330

(c) BY-NC-ND 
lahat ng gustong lumahok. Lamáng ang nakakaalam ng pasikut-sikot. Lamáng din hindi lang ang may nalalaman kundi kung sino ang kanyang kakilala. ${ }^{97}$ At iyong may pera at impluwensya ay lamáng sa pagkakataon, kakayahan, at maging sa motibasyon..$^{98}$

Mabuti ring usisain kung para ano, para kanino, at para saan ang pagsisipag at pagtitiyaga. Sa isang banda, nagsisikap at nagtitiyaga tayo para mabigyan ng magandang buhay ang ating pamilya; para maipagyabang natin sa ating mga sarili at sa iba na ginamit natin ng wasto ang ating talino, galing, at kadalubhasaan; para masabi natin sa ating mga sarili na narating natin ang tagumpay. Wala naman talagang masama rito. Kaya nga nakakahalina ang diskurso ng meritokrasya sapagkat nakabatay ito sa mga pagpapahalagang kagyat nating kinikilala at tinatanggap. Subalit sa sinasabi kong SMisasyon, ang modelo ng ganap na tagumpay ay mga taong nagtataguyod ng mga pagpapahalagang neoliberal. Nagsisikap at nagtitiyaga para $s a$ at $s a$ loob $n g$ isang sistema ng walang-katapusang kumpetisyon. Nagsisikap at nagtitiyaga para sukdulang makayapak at makasagasa. Nagsisikap at nagtitiyaga para maging epektibong turnilyo ng makina ng konsumeristang lipunan. Nagsisikap at nagtitiyaga para hindi maging titser lang kundi maging corporate man. Ganito ang pagkakasabi ni Littler: "Contemporary neoliberal discourses of meritocracy assume that all progressive movement must happen upwards and, in the process, contribute to the positioning of working-class cultures as the 'underclass', as abject zones and as lives to flee from." 99

\section{Pangwakas}

Ang SMisasyon ay pagpapalaganap ng kultura ng konsumerismo, pagtataguyod ng kultura ng matira-ang-matibay, at pagpapatatag ng mito ng sipag at tiyaga. Ang mga ito ay ilan lamang sa mga natatanging katangian ng neoliberalismo. Batid ko na ang sanaysay na ito ay isang munting hakbang sa isang mahaba-habang paglalakbay upang suriin ang mga bunga ng namamayaning ideolohiya sa ating lipunan.

Itong pag-usisa sa SMisasyon ay isang kakatwang gawain. Hindi ito madali sapagkat wala naman talaga sa labas itong sinusuri. At wala rin sa labas ang nagsusuri. Sa halina ng shopping mall, halos walang hindi maaakit. Sa kamandag ng mito ng meritokrasya, halos walang hindi mapaparalisa. Sa pananaig ng kultura ng matira-ang-matibay, halos walang hindi bibigay. Subalit kailangang ituloy ang gawain ng kritisismo. Kung saan at kailan

\footnotetext{
${ }_{97}$ Tingnan ang Stephen McNamee and Robert Miller, The Meritocracy Myth, 3rd ed. (Lanham: Rowman \& Littlefield, 2013).

${ }_{98}$ Arthur, Financial Literacy Education, 105.

${ }^{99}$ Littler, Against Meritocracy, 7.
} 


\section{SMISASYON NG LIPUNAN}

nagaganap ang dominasyon, doon din nagiging mas napapanahon ang pagtatanong, pag-iisip, pagsusuri at pagpupuna.

Mainam ding banggitin na wala akong nakikitang hangganan ng mga disiplina na susuri sa penomenon ng SMisasyon. ${ }^{100}$ Kailangang lusawin ang mga hangganan at hayaang tumawid at magsanib ang mga disiplina: pilosopiya, sosyolohiya, ekonomiks, pampulitikang agham, sikolohiya, cultural studies, media studies, kasaysayan, Philippine studies, postcolonialism, at marami pang iba. Wala ring masasabing gitna at laylayan sa mga ito. Ang lahat ay may potensiyal na mag-ambag ng iba't ibang natatanging pagtanaw sa neoliberalismo bilang SMisasyon sa karanasan, kaisipan, kamalayan, karakter, at kilos ng bawat Pilipino.

Department of Philosophy, University of Santo Tomas, Philippines

\section{References}

Anderson, Perry, "Renewals," New Left Review 1 (January to February 2000). Appleyard, Brian, "Shopping around for salvation: The new religion is consumerism and massive malls are its cathedrals. Let us bow our heads and pay," in The Independent (3 November 1993), $<\underline{\text { https://www.independent.co.uk/voices/shopping-around-for- }}$ salvation-the-new-religion-is-consumerism-and-massive-malls-areits-cathedrals-1501792.html $>$.

Arthur, Chris, Financial Literacy Education: Neoliberalism, the Consumer and the Citizen (Rotterdam: Sense Publishers, 2012).

Baudrillard, Jean, The Consumer Society: Myths and Structures (London: Sage Publications, 1998).

Bauman, Zygmunt, Consuming Life (Cambridge: Polity, 2007).

Bello, Walden, "EDSA, Neoliberalism, and Globalization" in Walden Bello (23 March 2017), <https://www.waldenbello.org/edsa-neoliberalismand-globalization/>.

"How neoliberalism killed the Philippines' EDSA Republic," in Green Left Weekly (24 June 2016), $<$ https://www.greenleft.org.au/content/walden-bello-howneoliberalism-killed-philippines-edsa-republic $>$.

"Neoliberalism as Hegemonic Ideology in the Philippines: Rise, Apogee, and Crisis," in Focus on the Global South (18 October 2009), $<$ https://focusweb.org/neoliberalism-as-hegemonic-ideology-in-thephilippines-rise-apogee-and-crisis/>.

100 Tingnan ang Henry Giroux, Border crossings: Cultural workers and the politics of education, 2nd ed. (New York: Routledge, 2005).

(c) 2019 Franz Giuseppe F. Cortez

https://www.kritike.org/journal/special issue/cortez april2019.pdf

ISSN 1908-7330

(cc) BY-NC-ND 
Bertman, Stephen, Hyperculture: Human Cost of Speed (Westport: Praeger, 1998).

Billig, Michael, "Commodity Fetishism and Repression: Reflections on Marx, Freud and the Psychology of Consumer Capitalism," Theory and Psychology, 9:3 (1999).

Bloodworth, James, The Myth of Meritocracy: Why Working-Class Kids Get Working-Class Jobs (London: Biteback Publishing, 2016).

Bourdieu, Pierre, Acts of Resistance: Against the Tyranny of the Market, trans. by Richard Nice (New York: The New Press, 1998).

Broad, Robin and John Cavanagh, Preface to Unequal Alliance: The World Bank, the International Monetary Fund, and the Philippines (Berkeley: University of California Press, 1988).

"Reframing Development in the Age of Vulnerability: From Case Studies of the Philippines and Trinidad to New Measures of Rootedness," Third World Quarterly, 32:6 (2011).

Bryman, A. E., "The Disneyization of Society," The Sociological Review, 47: 1 (1999).

Clarke, Simon, "The Neoliberal Theory of Society," in Neoliberalism: A Critical Reader, ed. by Alfredo Saad-Filho \& Deborah Johnston (London: Pluto, 2005).

de Castro, Julio Cesar, "The Consumer as Agent in Neoliberalism," Matriz, 9:2 (2015).

Dean, Jodi, "Enjoying Neoliberalism," Cultural Politics, 4:1 (2008).

Dela Paz, Chrissee, "Fast Facts: SM Investments Corporation," Rappler (28 June 2017), <https://www.rappler.com/business/170638-fast-factssm-investments-corporation-henry-sy-malls $>$.

Duncan, Aaron, Gambling with the Myth of American Dream (New York: Routledge, 2015).

Dunn, Robert, Identifying Consumption: Subjects and Objects in Consumer Society (Philadelphia: Temple University Press, 2008).

Francis, Evangelii Gaudium, Apostolic Exhortation (24 November 2013), <http://w2.vatican.va/content/francesco/en/apost exhortations/docu ments/papa-francesco esortazione-ap 20131124 evangeliigaudium.html>.

Giroux, Henry, Giroux, Border crossings: Cultural workers and the politics of education, 2nd ed. (New York: Routledge, 2005).

"Neoliberal Fascism and the Echoes of History," in Truthdig (2 August 2018), <https://www.truthdig.com/articles/neoliberalfascism-and-the-echoes-of-history/>.

Gonzales, Iris, "SM Prime eyes 75 malls by 2018," in Philippine Star (2 September 2015), <https://www.philstar.com/business/2015/09/02/ 1495238/sm-prime-eyes-75-malls-2018>.

(C) 2019 Franz Giuseppe F. Cortez

https://www.kritike.org/journal/special issue/cortez april2019.pdf

ISSN 1908-7330 


\section{6}

SMISASYON NG LIPUNAN

Guiltinan, Joseph, "Creative Destruction and Destructive Creations: Environmental Ethics and Planned Obsolescence," Journal of Business Ethics, 89 (2009).

Harvey, David, A Brief History of Neoliberalism (Oxford: Oxford University Press, 2005).

Hedman, Eva-Lotta and John Sidel, Philippine Politics and Society in the Twentieth Century: Colonial Legacies, Postcolonial Trajectories (London: Routledge, 2000).

"Henry Sy," in "2018 Philippines 50 Richest Net Worth," in Forbes (as of 5 September 2018), <https://www.forbes.com/profile/henrysy/?list=philippines-billionaires\#2cec94e14a10>.

"Henry Sy tops richest Filipinos list for 10th year in a row - Forbes," in CNN Philippines (25 August 2017), <http://nine.cnnphilippines.com/ business/2017/08/24/Forbes-riches-Filipinos.html >.

Hindle, Tim, The Economist Guide to Management Ideas and Gurus (London: Profile Books, 2008).

"How SM won with turon," in SM Investments Corporation (30 May 2017), $<$ https://www.sminvestments.com/company-releases/how-sm-wonturon>.

Jones, Campbell, Rene ten Bos, and Martin Parker, For Business Ethics: A Critical Approach (London: Routledge, 2005).

Jones, Daniel, Masters of the Universe: Hayek, Friedman and the Birth of Neoliberal Politics (New Jersey: Princeton University Press, 2012).

Karnani, Aneel, "Romanticizing the Poor," Stanford Social Innovation Review (Winter 2009).

Khandizaji, Amirhosein, Baudrillard and the Culture Industry: Returning to the First Generation of the Frankfurt School (Switzerland: Springer Nature, 2017).

Klein, Naomi, The Shock Doctrine: Rise of Disaster Capitalism (New York: Metropolitan, 2007).

Larner, Wendy, "Neo-liberalism: Policy, Ideology, Governmentality," Studies in Political Economy, 63:1 (Autumn 2000).

Lawn, Jennifer and Chris Prentice, "Introduction: Neoliberal Culture/The Cultures of Neoliberalism," Sites: New Series, 12:1 (2015).

Lea, David, "Darwinism and Ethics," in Encyclopedia of Business Ethics, ed. by Robert Kolb (Los Angeles: Sage Publications, 2008).

Littler, Jo, Against Meritocracy: Culture, Power and Myths of Mobility (London: Routledge, 2018).

"Meritocracy as Plutocracy: The Marketising of Equality within Neoliberalism," New Formations: A Journal of Culture/Theory/Politics, 80-81 (2013), 52-72.

(c) 2019 Franz Giuseppe F. Cortez

https://www.kritike.org/journal/special issue/cortez april2019.pdf

ISSN 1908-7330

(c) $)$ BY-NC-ND 
Lindio-McGovern, Ligaya, "Neoliberal Globalization in the Philippines: Its Impact on Filipino Women and Their Forms of Resistance," Journal of Developing Societies, 23:1-2 (2007).

Madik, Vladimir, Aaron Ahuvia, and Elif Izberk-Bilgin, "Ebayization," in The Blackwell Encyclopedia of Sociology, ed. by George Ritzer (USA: Blackwell, 2007).

Marx, Karl, "The Fetishism of the Commodity and Its Secret," in Capital: A Critique of Political Economy, Vol. 1, trans. by Ben Fowkes (Middlesex, England: Penguin Books, Ltd., 1976).

McCarthy, J., "Privatizing Conditions of Production: Trade Agreements as Neoliberal Environmental Governance," in Neoliberal Environments: False Promises and Unnatural Consequences, ed. by N. Heynen, J. McCarthy, S. Prudham, and P. Robbins (London: Routledge, 2007).

McChesney, Robert, Introduction to Noam Chomsky, Profit over People: Neoliberalism and Global Order (New York: Seven Stories, 1999).

McNamee, Stephen and Robert Miller, The Meritocracy Myth, 3rd ed. (Lanham: Rowman \& Littlefield, 2013).

Miles, Steven, Consumerism as a Way of Life (London: Sage, 1998).

Mirowski, Philip, "Postface: Defining Neoliberalism," in The Road from Mont Pelerin: The Making of Neoliberal Thought Collective, ed. by Philip Mirowski and Dieter Plehwe (Cambridge: Harvard University Press, 2009).

Mitchell, Timothy, "How Neoliberalism Makes Its World," in The Road from Mont Pelerin: The Making of Neoliberal Thought Collective, ed. by Philip Mirowski and Dieter Plehwe (Cambridge: Harvard University Press, 2009).

Monbiot, George, "Neoliberalism - the ideology at the root of all our problems," in The Guardian (15 April 2016), $<$ https://www.theguardian.com/books/2016/apr/15/neoliberalismideology-problem-george-monbiot>.

Oliveros, Benjie, "Neoliberalism and greater Inequality," Bulatlat: Journalism for the people (9 June 2016), <https://www.bulatlat.com/2016/06/09/ neoliberalism-and-greater-inequality/>.

Peet, Richard, Unholy Trinity: The IMF, World Bank and WTO, 2nd ed. (London: Zed Book, 2003).

Reed, Daryl, Peter Utting, and Ananya Mukherjee-Reed, eds., Business Regulations and Non-State Actors: Whose Standards, Whose Development? (London: Routledge, 2012).

"Rehashed neoliberal policies: One year of Dutertenomics," in Ibon (28 June 2017), < https://www.ibon.org/rehashed-neoliberal-policies-one-yearof-dutertenomics/>.

(C) 2019 Franz Giuseppe F. Cortez https://www.kritike.org/journal/special issue/cortez april2019.pdf ISSN 1908-7330 
Rey, Aika, "Malacañang: Henry Sy Sr a 'pillar of Philippine economy'," in Rappler (19 January 2019), <https://www.rappler.com/nation/221351malacanang-statement-henry-sy-sr-pillar-philippine-economy>.

Rico, Jore-Annie and Kim Robert C. de Leon, "Mall culture and consumerism in the Philippines," in State of Power 2017, ed. by Nick Buxton and Deborah Eade (Amsterdam, Netherlands: Transnational Institute, 2017), <https://www.tni.org/files/publicationdownloads/stateofpower2017-mall-culture.pdf $>$.

Rimando, Lala, "Forbes Philippines: Reinventing the mall," in SM Prime (8 April 2016), <https://smprime.com/media/news/forbes-philippinesreinventing-mall>.

Ritzer, George, "The McDonaldization of Society," The Journal of American Culture 6 (1983).

The McDonaldization of Society: An Investigation into the Changing Character of Contemporary Social Life (California: Pine Forge, 1993).

Ritzer, George, Douglas Goodman, and Wendy Wiedenhoft, "Theories of Consumption," in Handbook of Social Theory, ed. by George Ritzer and Barry Smart (London: Sage, 2001)

Saad-Filho, Alfredo and Deborah Johnston, Introduction to Neoliberalism: A Critical Reader, ed. by Alfredo Saad-Filho \& Deborah Johnston (London: Pluto, 2005).

Said, Edward, "Problems of Neoliberalism," in ZNet (20 September 2000), $<$ https://zcomm.org/zcommentary/problems-of-neoliberalism-byedward-said/>.

Slade, Giles, Made to Break: Technology and Obsolescence in America (USA: Harvard University Press, 2006).

"SM Prime to open 7 new malls in 2017," in Rappler (30 August 2016), $<$ https://www.rappler.com/business/industries/175-realestate/144625-sm-prime-new-malls-2017>.

Steger, Manfred and Ravi Roy, Neoliberalism: A Very Short Introduction (Oxford: Oxford University Press, 2010).

Sugarman, Jeff, "Neoliberalism and Psychological Ethics," in Journal of Theoretical and Philosophical Psychology, 35:2 (1995).

Tan, Michael L., "'Sandamakmak'," in Inquirer Opinion (15 August 2018), $<$ https://opinion.inquirer.net/115379/sandamakmak $>$.

Tejero, Constantino, "Iloilo: Discover its history, decode its modernity," in Inquirer Lifestyle (11 February 2017), $<\underline{\text { https://lifestyle.inquirer.net/254095/iloilo-discover-history-decode- }}$ modernity/>.

Tolentino, Rolando, "Kabataang Katawan, Mall at Siyudad: Mga Tala sa Gitnang Uring Karanasan at Neoliberalismo" Philippine Humanities Review, 14:12 (April 2012).

(c) 2019 Franz Giuseppe F. Cortez

https://www.kritike.org/journal/special issue/cortez april2019.pdf

ISSN 1908-7330

(c) BY-NC-ND 
Kulturang Mall (Manila: Anvil, 2004).

"Mall, malling, nagmo-malling...," in Pinoy Weekly (23 January 2010), <https://www.pinoyweekly.org/2010/01/mall-malling-nagmomalling\%e2\%80\%a6/>.

- Sa Loob at Labas ng Mall Kong Sawi / Kaliluha'y Siyang Nangyayaring Hari: Ang Pagkatuto at Pagtatanghal ng Kulturang Popular (Quezon City: University of the Philippines, 2001).

"Syudad ng malls, Kulturang Popular," in Rolando Tolentino (21 April 2009), <https://rolandotolentino.wordpress.com/2009/04/21/ syudad-ng-malls-kulturang-popular-kultura-column-bulatlatcom/>.

Wagnleitner, Reinhold, Coca-Colonization and the Cold War: The Cultural Mission of the United States in Austria after the Second World War (London: University of North Carolina, 1994).

Ward, Kevin and Kim England, "Introduction: Reading Neoliberalization," in Neoliberalization: States, Networks, Peoples, ed. by Kim England and Kevin Ward (USA: Blackwell, 2007). 